\title{
How to Deal with A Severe Dry Syndrome (Case Report)
}

\author{
El Baroudi Taieb", Belghmaidi Sarah, Hajji Ibtissam, Moutaouakil Abdeljalil
}

Department of Ophtalmology, University Hospital Center Mohammed VI, Marrakesh, Morocco

DOI: $\underline{10.36347 / \mathrm{sasjs} .2020 . \mathrm{v} 06 \mathrm{i} 05.005}$

| Received: 09.05.2020 | Accepted: 16.05.2020 | Published: 19.05.2020

*Corresponding author: El Baroudi Taieb

Abstract

Case Report

Introduction: Sjögren's syndrome (SS) is a relatively common autoimmune disease. It affects 0.2 to $3 \%$ of the population. For many years, the treatment of SS has remained mainly symptomatic, the doctors do not have drugs really capable of influencing the course of this disease, especially with regard to dry eye syndrome. The interest of this clinical case is to expose the complexity of the care of a patient presenting a severe dry eye syndrome and the therapeutic challenges that the ophthalmologist makes. Case presentation: M.T 65-year-old man, followed for 15 years in internal medicine for mixed connective tissue disease. The evolution was marked by the appearance of macular toxicity to A synthetic antimalarials, which required the discontinuation of treatment followed by a significant agravation of the dry eye syndrome and the ocular symptomatology. His care had involved a therapeutic adaptation and amniotic membrane graft and especially a fitting of a scleral lens. Conclusion: The treatment of dry eye is complex, difficult to conduct because of the multitude of mechanisms and the low reliability of the diagnostic criteria, as well as the frequent discordance between signs and symptoms. Treatment must first and foremost be based on a fine analysis of the mechanisms underlying dry eye and try to involve the patient in his treatment process.

Keywords: Sjögren's syndrome (SS), symptomatic, dry eye syndrome, antimalarials.

Copyright @ 2020: This is an open-access article distributed under the terms of the Creative Commons Attribution license which permits unrestricted use, distribution, and reproduction in any medium for non-commercial use (NonCommercial, or CC-BY-NC) provided the original author and source are credited.

\section{INTRODUCTION}

Sjögren's syndrome (SS) is a relatively common autoimmune disease. It affects 0.2 to $3 \%$ of the population. For many years, the treatment of SS has remained mainly symptomatic, the doctors do not have drugs really capable of influencing the course of this disease, especially with regard to dry eye syndrome [1].

The interest of this clinical case is to expose the complexity of the care of a patient presenting a severe dry eye syndrome and the therapeutic challenges that the ophthalmologist makes.

\section{CASE PRESENTATION}

M.T 65-year-old man, followed for 15 years in internal medicine for mixed connective tissue disease (Sjogren Syndrome + systemic lupus erythematosus) with bilateral and inaugural ocular involvement. During these 15 years, the patient was put under general treatment based on synthetic antimalarials (hydroxychloroquine) and lacrimal substitute based on sodium hyaluronate with conservative (benzalkonium). The evolution was marked by the appearance of macular toxicity to A synthetic antimalarials, which required the discontinuation of treatment followed by a significant agravation of the dry eye syndrome and the ocular symptomatology.

In front of this clinical aggravation the patient was sent to our service for specialized care.

\section{First Consultation}

- The initial examination of the patient finds a very photophobic patient, with that palpebral opening almost impossible.

- The National Eye Institute Visual Function Questionnaire-25 (NEI-VFQ25) find a score at 24 and the Ocular Surface Disease Index (OSDI) to 64.

- Surface examination reveals conjunctival fibrosis, Filamentary Keratitis and a severe Superficial Punctate Keratitis, the Break-up time (BUT) was under 10 seconde (3 s) Shirmer test $2 \mathrm{~mm} / 5 \mathrm{~min}$ (Figure-1).

- We decided to stop eye drops with preservative, prescribe eye drops without preservatives (sodium hyaluronate) with instillation hourly, ointment at night containing triglycerides to rebalance the lipid composition of tears and fight against evaporation, eyelid hygiene. 


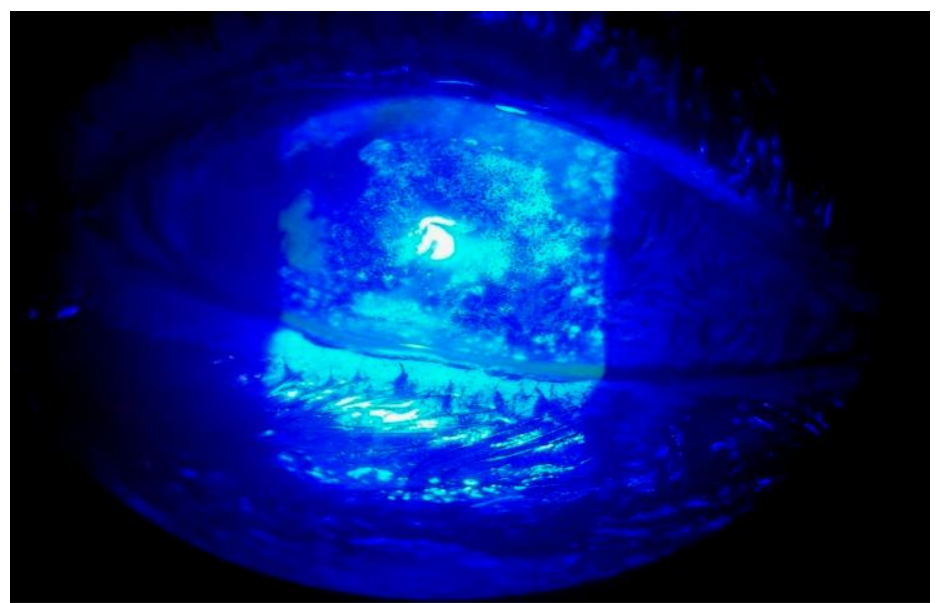

Fig-1: The initial examination of the patient finding a Filamentary Keratitis and a severe Superficial Punctate Keratitis

Month 1

- The National Eye Institute Visual Function Questionnaire-25 (NEI-VFQ25) find a score at 35 and the Ocular Surface Disease Index (OSDI) to 58 .

- Surface examination reveals a small improvement with regression of the Filamentary Keratitis and a the Superficial Punctate Keratitis with a BUT always altered.

- The therapeutic decision was to keep the same treatment and to put punctal plugs into tear ducts to block drainage.

Month 2

- We found no change in the surface examination and the patient was always sympomayological (The National Eye Institute Visual Function Questionnaire-25 (NEI-VFQ25) and the Ocular Surface Disease Index (OSDI) always to 35 and 58 respectively ).
- In front of this situation a joint decision with the internists staff to start a biological therapies the rituximab an anti-CD20 monoclonal antibody, eye drops containing cyclosporine $0.05 \%$ and autologous serum eye drops.

Month 3

- For the first time we note a considerable improvement in the state of the ocular surface (regression of the Filamentary Keratitis and a the Superficial Punctate Keratitis and an improvement of the BUT 8 seconde) (The National Eye Institute Visual Function Questionnaire-25 (NEI-VFQ25) and the Ocular Surface Disease Index (OSDI) 48 and 40 respectively) (Figure-2).

- In view of these encouraging results we decided to keep the patient under the same treatment (rituximab an anti-CD20 monoclonal antibody, cyclosporine $0.05 \%$ and autologous serum eye drops, sodium hyaluronate eye drops and ointment at night).

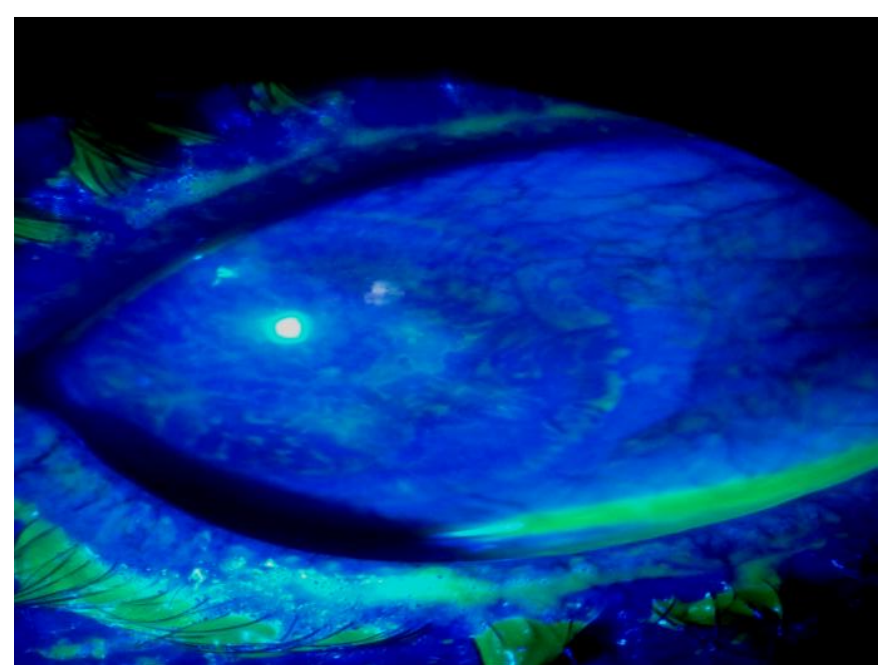

Fig-2: Photo taken in the third month, after starting a treatment based on a biological therapies the rituximab an anti-CD20 monoclonal antibody, eye drops containing cyclosporine $0.05 \%$ and autologous serum eye drops. which shows a considerable improvement in the state of the ocular surface (regression of the Filamentary Keratitis and a the Superficial Punctate Keratitis 
Month 7

- After several months of good clinical evolution, the evolution was marked by the appearance of a paracentral nasal preoperative corneal ulcer $(4 \mathrm{~mm} * 2.9 \mathrm{~mm})$.

- In front of this urgent situation, threatening the visual and anatomical prognosis of the eye an amniotic membrane (A.M) transplant has been done.

- During this critical period, we noticed a deterioration of questionnaires NEI-VFQ25 and OSDI 28 and 64 respectively.
Month 11

- Fortunately, the evolution was favorable, a complete healing of the ulcer was obtained after 1 month at the expense of a scar on the cornea.

- Next to the general treatment we decided to adapt the patient with large diameter scleral lenses and replace the sodium hyaluronate eye drops by a new eye drops containing trealose (3 $\mathrm{g}$ for $100 \mathrm{~mL}$ ) and sodium hyaluronate $(0,15 \mathrm{~g}$ pour $100 \mathrm{~mL})$ (Figure-3).

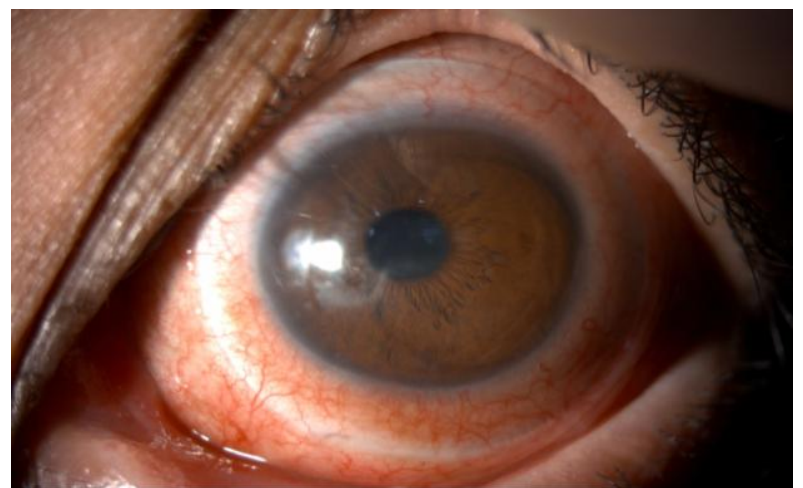

Fig-3: Picture taken at the eleventh month showing complete healing of the ulcer at the expense of a scar on the cornea and the improvement of the state of the surface and ocular functional signs due to general treatment and the large diameter scleral lenses and the new eye drops containing trealose + sodium hyaluronate

\section{Month 12}

- Due to general treatment and the large diameter scleral lenses and the new eye drops containing trealose + sodium hyaluronate, we constated an improvement of the state of the surface and ocular functional signs.

- An improvement of the dry eye syndrome questionnaires was also reported NEI-VFQ25 and OSDI were 56 and 28 respectively.

Month 18

- The patient was able to regain a social and professional activity
- The National Eye Institute Visual Function Questionnaire-25 (NEI-VFQ25) find a score at 86 and the Ocular Surface Disease Index (OSDI) to 24 .

Graph 4 summarizes the evolution of the questionnaires (NEI-VFQ25) and the Ocular Surface Disease Index (OSDI) throughout the months of treatment and the treatment received by the patient.

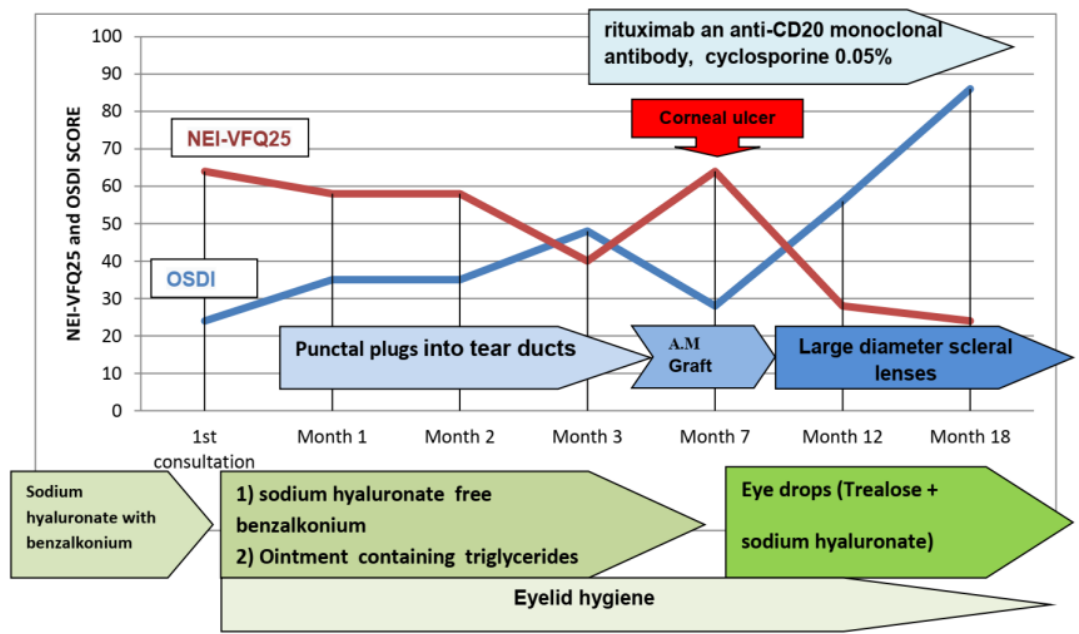

Graph-4: Summarizing the evolution of the questionnaires (NEI-VFQ25) and the Ocular Surface Disease Index (OSDI) throughout the months of treatment and the treatment received by the patient 


\section{DISCUSSION}

Treating a dry eye is always difficult, complex and often frustrating, despite the fact that the therapeutic means are multiple. Even the expected objectives are ambiguous:

- Suggest a symptomatic treatment or cure the surface?

- Reduce pain and improve quality of life?

If the dry eye was limited to a simple lack of water on the surface of the eye, it would be simple to treat it and relieve the patients by banal tears artificial, but unfortunately the mechanisms underlying drought are complex and intricate. All ophthalmologists know how difficult it is to treat and relieve these patients, even more definitely to cure the disease, and how often the therapies are disappointing, and often they face lack of systematic correlation between eye irritation symptoms and clinical tests, assessing the severity of dry eye can be problematic [2].

The evaluation of the severity of dry eye is a fundamental element to define the therapeutic strategy $[3,4]$, so it is necessary to use standardized questionnaires to be able to classify drought and evaluate the efficacy of the proposed treatments. In our case we chose The National Eye Institute Visual Function Questionnaire-25 (NEI-VFQ25) and the Ocular Surface Disease Index (OSDI).

The choice of treatment will be guided by the severity and means available. The logical basis of dry eye treatments is based primarily on tear substitutes, without forgetting to act on the environment, dry and air-conditioned environments, posture problems during prolonged computer work, are all sources of visual fatigue and drought, both are very often entangled [5]. The removal of the preservative is essential in the case of dry eye and help these patients to find the most suitable time and the most suitable products for their symptoms. It is often interesting to offer products with different properties and viscosities and let patients find the most appropriate pattern for themselves.

Besides lacrimal substitutes, we have to make sure that the tear film is concerved. That is possible by using punctal plugs into tear ducts [6]. This perfectly logical treatment in theory often has the disadvantage of retaining highly inflammatory tears, which can make efficiency insufficient or disappointing. Thus, the reduction of inflammation is a real challenge to try to get out of the vicious circle of the disease. Ciclosporin at very low concentrations $(0.05$ to $0.1 \%)$ much lower than the $2 \%$ usually used for the prevention and treatment of graft rejection, has been shown to be effective in dry keratoconjunctivitis with an inflammatory component [7], reducing symptoms, keratitis and inflammation $[8,9]$.
In case of visual threat, by an ulcer for example, as this is the case with our patient, autologous serum, amniotic membrane can be interesting alternatives [10, 11].

Scleral lenses constitute a very effective physical means in the treatment of cases of severe sechresse, they provide real relief and an improvement in visual acuity [12].

The emergence of biological therapies finally suggests the possibility of influencing the course of this disease and being able to cure it. Molecules that target B cells, such as rituximab, an anti-CD20 antibody, or epratuz umab, an anti-CD22 antibody, have already shown significant results [13].

\section{CONCLUSION}

The treatment of dry eye is complex, difficult to conduct because of the multitude of mechanisms and the low reliability of the diagnostic criteria, as well as the frequent discordance between signs and symptoms. Treatment must first and foremost be based on a fine analysis of the mechanisms underlying dry eye and try to involve the patient in his treatment process

\section{REFERENCES}

1. Rossier V, Bart PA, Spertini F. Syndrome de Sjögren: enfin une nouvelle approche de traitement [Sjögren's syndrome: a new approach to treatment]. Revue médicale suisse. 2012;8(337):843-7.

2. Baudouin C, Aragona P, Van Setten G, Rolando M, Irkeç M, del Castillo JB, Geerling G, Labetoulle M, Bonini S. Diagnosing the severity of dry eye: a clear and practical algorithm. British Journal of Ophthalmology. 2014 Sep 1;98(9):1168-76.

3. Behrens A, Doyle JJ, Stern L, Chuck RS, McDonnell PJ, Azar DT, Dua HS, Hom M, Karpecki PM, Laibson PR, Lemp MA. Dysfunctional tear syndrome: a Delphi approach to treatment recommendations. Cornea. 2006 Sep 1;25(8):900-7.

4. Pflugfelder SC, Geerling G, Kinoshita S, Lemp MA, McCulley JP, Nelson D, Novack GN, Shimazaki J, Wilson C. Management and therapy of dry eye disease: report of the Management and Therapy Subcommittee of the International Dry Eye WorkShop (2007). Ocular Surface. 2007 Apr;5(2):163-78.

5. Uchino M, Uchino Y, Dogru M, Kawashima M, Yokoi N, Komuro A, Sonomura Y, Kato H, Kinoshita S, Schaumberg DA, Tsubota K. Dry eye disease and work productivity loss in visual display users: the Osaka study. American journal of ophthalmology. 2014 Feb 1;157(2):294-300.

6. Bourkiza R, Lee V. A review of the complications of lacrimal occlusion with punctal and canalicular plugs. Orbit, 2012; 2:86-93. 
7. Schultz C. Safety and efficacy of cyclosporine in the treatment of chronic dry eye. Ophthalmology and Eye Diseases, 2014;6:37-42.

8. Brignole F, Pisella PJ, De Saint Jean M, Goldschild M, Goguel A, Baudouin C. Flow cytometric analysis of inflammatory markers in KCS: 6-month treatment with topical cyclosporin A. Investigative ophthalmology \& visual science. 2001 Jan 1;42(1):90-5.

9. Brignole F, Pisella PJ, Goldschild M, De Saint Jean M, Goguel A, Baudouin C. Flow cytometric analysis of inflammatory markers in conjunctival epithelial cells of patients with dry eyes. Investigative ophthalmology \& visual science. 2000 May 1;41(6):1356-63.

10. Creuzot-Garcher C, Lafontaine PO, Brignole F, Pisella PJ, d'Athis P, Bron A, Lapierre V,
Baudouin C. Treating severe dry eye syndromes with autologous serum. Journal francais d'ophtalmologie. 2004 Apr;27(4):346-51.

11. Datta H, Sarkar K, Chatterjee PR. Amniotic membrane transplantation in ocular surface disorders. Journal of the Indian Medical Association 2004;12:726-9.

12. Segal O, Barkana Y, Hourovitz D, Behrman S, Kamun Y, Avni I, Zadok D. Scleral contact lenses may help where other modalities fail. Cornea. 2003 May 1;22(4):308-10.

13. Dass S, Bowman SJ, Vital EM, Ikeda K, Pease CT, Hamburger J, Richards A, Rauz S, Emery P. Reduction of fatigue in Sjögren syndrome with rituximab: results of a randomised, double-blind, placebo-controlled pilot study. Annals of the rheumatic diseases. 2008 Nov 1;67(11):1541-4. 